\title{
基于成本效益重要度的复杂系统可靠性保障策略
}

\section{王成 ${ }^{1}$, 许建新 $^{2}$, 张振明 $^{2}$, 王红军 ${ }^{3}$}

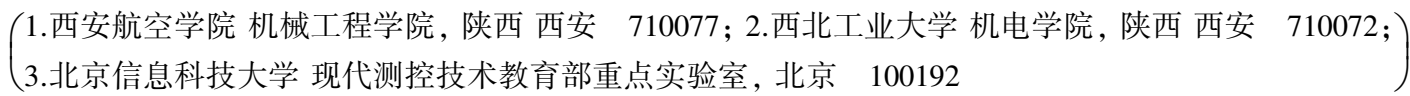

摘 要: 为了保障复杂系统的长期稳定经济运行, 对系统运行过程和需要解决的问题进行了描述, 构 建了系统可靠性模型和组件更换优化模型,采用边际效应思想和重要度理论,提出了一种基于成本效 益重要度的复杂系统可靠性保障策略, 旨在寻求以系统可靠度下限阈值和运行时长为约束、以维护成 本最小为目标的组件预防更换序列。当系统可靠度下降到预设阈值时,计算系统各组件的成本效益 重要度,选择成本效益重要度最大的组件进行更换, 如此进行迭代, 直到完成运行任务, 形成最优的组 件更换序列。以可等效为串并联系统的复杂系统为例验证了该策略的可行性。该策略对保障一些高 端装备安全关键系统的可靠运行具有一定的参考意义。

\section{关 键 词:复杂系统; 可靠性保障;成本效益重要度;边际效应;预防更换}

中图分类号:TH17

文献标志码: A

文章编号: 1000-2758(2020)06-1316-06
维护在工业生产和系统安全领域扮演着重要的 作用,特别是一些因系统失效而产生巨大损失的领 域。已有多种维护策略被用于提升系统可靠性、减 少系统失效和生产成本 ${ }^{[1]}$ 。对于不可维修系统, 常 采用预防更换 (preventive replacement, PR) 的方式 来保障系统的可靠性。预防更换是指当系统仍在运 行时, 对系统或包含的组件进行更换的一种策 略 ${ }^{[2]}$ 。常见的预防更换多用于单一组件系统 ${ }^{[3]}$ 、多 个相同组件组成的系统 ${ }^{[4]}$ 、或由多个不同组件组成 的复杂系统的一次更换过程 ${ }^{[5]}$, 而涉及复杂系统多 次更换过程的研究并不多见。

近年来, 由于生产系统的复杂性和多样性日益 增加, 由许多不同组件构成的复杂退化系统的可靠 性保障受到了更加广泛的关注 ${ }^{[5]}$ 。由于每个组件 对系统有着各自的贡献, 因此, 选择最重要组件作为 更换对象对确保系统的可靠性至关重要。重要度 (importance measures, IM $)^{[6]}$ 被广泛应用于系统工 程、可靠性工程、复杂系统等领域, 用以评估各种对 象的相对重要程度。关于 IM, 学者们已经进行了大 量的研究工作, 常用的有 Birnbaum 重要度 (birnbaum importance, $\mathrm{BI})^{[7]}$ 、关键重要度 ${ }^{[8]}$ 、综合
重要度 ${ }^{[9]}$ 和成本重要度 ${ }^{[10]}$ 等,有关 IM 的最新研究 进展请参阅文献 [11-12]。

鉴于 IM 的优势, 本文对系统运行过程和需要 解决的问题进行了描述, 构建了系统可靠性模型和 组件更换优化模型,采用边际效应方法和重要度理 论, 提出了一种基于成本效益重要度的复杂系统可 靠性保障策略, 旨在寻求保障系统可靠度和运行时 长的前提下实现维护成本最小的组件预防更换 序列。

\section{1 问题描述}

考虑一个由 $n$ 个不同组件构成的复杂系统, 其 中每个组件都有其自身的退化过程, 系统可靠度取 决于组件的可靠度。在一次任务中, 要求系统的可 靠度不低于预设國值。为了保障系统的长期可靠运 行,需要在运行周期内进行多次维护并且使维护成 本达到最小。整个过程的描述如下: 当系统可靠度 下降到预设阈值时, 进行第一次 PR 动作, 以此类 推, 共进行 $k$ 次 PR 动作, 直到运行任务中止。在组 件更换过程中,由于每次仅选择最需要的组件进行 
更换, 系统在运行期间的整体可靠度常常小于 1 。 在一次任务执行中, 系统可靠度随时间和组件更换 而变化的规律如图 1 所示。本文所要解决的问题是 在保障系统可靠度不低于预设阈值以及运行时长的 前提下, 寻找一组组件更换序列使整个系统的维护 成本最小。

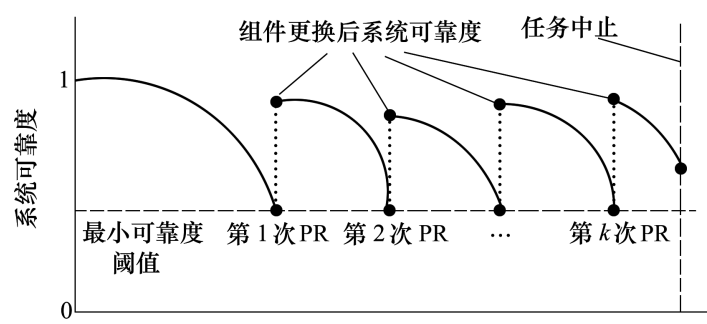

任务运行时间 / 月

图 1 系统可靠度随时间和组件更换变化规律示意图

为了保证研究的有效性,特作如下假设:

1) 组件之间相互独立, 每个组件的退化水平都 能被检测到;

2) 退化是引起组件失效的唯一原因, 不考虑如 人为损坏、自然灾害等外部环境对组件的影响;

3) 当系统在运行中暂停时, 系统不会发生 退化。

\section{2 系统可靠性建模}

本文中考虑的复杂系统是一个能够等效成由多 个不同组件组成的串并联系统。每个组件对系统都 有自己的贡献, 系统的可靠度由这些组件的可靠度 来衡量。组件的失效程度由其退化程度来衡量, 每 个组件的退化机理都遵循自己的退化路径。定义组 件 $i$ 随着时间 $t$ 的退化水平表示为 $X_{i}\left(t ; \mu_{i}, \varepsilon_{i}\right)$, 其中 $\mu_{i}$ 表示退化率; $\varepsilon_{i}$ 表示随机影响参数且服从正态分 布, 即 $\varepsilon_{i} \sim N\left(0, \sigma_{i}^{2}\right)$ 。在大多数情况下, $X_{i}\left(t ; \mu_{i}\right.$, $\left.\varepsilon_{i}\right)$ 是一个随着时间 $t$ 变化的单调函数 ${ }^{[13-14]}$ 。

对于每一个组件当其退化水平 $X_{i}$ 超过预设阈 值 $L_{i}$ 时, 则认为该组件失效。所有组件预设失效阈 值的集合为 $\boldsymbol{L}=\left\{L_{1}, L_{2}, \cdots, L_{n}\right\}$ 。不失一般性, 假设 组件退化水平为单调增, 在 $t$ 时刻组件 $i$ 的可靠度由 $X_{i}$ 不超过 $L_{i}$ 的概率来表示, 即

$$
\begin{gathered}
R_{i}(t)=\operatorname{Pr}\left\{X_{i}\left(t ; \mu_{i}, \varepsilon_{i}\right)<L_{i}\right\}= \\
\operatorname{Pr}\left\{\mu_{i} t+\varepsilon_{i}<L_{i}\right\}=\Phi\left(\frac{L_{i}-\mu_{i} t}{\sigma_{i}}\right)
\end{gathered}
$$

式中: $R_{i}(t)$ 为第 $i$ 个组件的可靠度函数; $\Phi(\cdot)$ 为标 准正态累积分布函数。

对于由 $m$ 个子系统组成的串并联系统, 每个子 系统包含 $n_{i}$ 个组件, 整个系统可靠度 $R(t)$ 可以表 示为

$$
R(t)=\prod_{i=1}^{m}\left(1-\prod_{j=1}^{n_{i}}\left(1-R_{i j}(t)\right)\right)
$$

式中: $m$ 为子系统的数量; $n_{i}$ 为每个子系统包含组件 的数量, 且 $i \in\{1,2, \cdots, m\} ; R_{i j}(t)$ 为第 $i$ 个子系统 的第 $j$ 个组件的可靠度。

\section{3 可靠性保障策略}

\section{1 目标函数定义}

将系统可靠度下限阈值 $\sigma$ 和规定的运行周期 $S$ 作为约束条件, 当系统可靠度低于 $\sigma$ 时进行组件更 换, 此时更换时刻可表示为 $T_{j}=\arg \inf \{t: R(t) \leqslant \sigma$; $\left.t>T_{j-1}, j=1,2, \cdots, k\right\}$, 其中, $T_{j}$ 表示第 $j$ 次更换时刻 且 $T_{0}=0 ; k$ 表示总更换次数且 $k=\max \left\{m \mid \sum_{j=1}^{m}\right.$ $\left.\left(T_{j}-T_{j-1}\right) \leqslant S\right\}$ 。组件的更换原则为在整个运行周 期内使其更换总成本达到最小, 即

$$
C=\min \sum_{i=1}^{n} e_{i} c_{i}
$$

式中: $C$ 为系统运行周期内组件更换最小总成本; $n$ 为系统组件的种类数量; $i$ 为组件编号, 且 $i \in\{1,2$, $\cdots, n\} ; e_{i}$ 为第 $i$ 个组件更换的数量 $; c_{i}$ 为第 $i$ 个组件 的更换成本。

为了确定最优的组件更换序列, 模拟整个周期 内组件消耗过程来反向求解各组件的更换时刻。由 于在整个模拟运行周期内会经历多次组件的更换工 作, 每一次仅考虑更换一个组件, 也就是说, 已有的 $n$ 个组件进行更换的机率是相同的。现在的难点 是, 在每一次更换中都有 $n$ 种可能, 而这 $n$ 种可能都 会产生 $n$ 种下次不同的更换时刻, 以此类推, 如果整 个过程进行 $k$ 次更换的话, 就有 $n^{k}$ 种组合, 在这么多 组合中寻找最优的组合难度相当大。因此, 需要寻 找新的方法进行求解。

\section{2 成本效益重要度}

本文的组件更换序列属于非线性整数规划问 题, 使用一般的规划论方法求解, 过程较为复杂, 因 此采用边际效应思想进行优化求解, 以达到对有效 资源的合理利用。为此, 将周期内组件更换的最小 
成本问题转化为在任意一次更换时刻选择哪一个组 件进行更换才能使所选择的组件单位成本提升的系 统可靠度增量最大。

为了便于研究, 取第 $j$ 次组件更换时刻作为研 究时刻点, 求出该时刻单位成本提升的系统可靠度 增量。在第 $j$ 次更换时刻对第 $i$ 个组件进行更换后 单位成本提升的系统可靠度增量可以表示为

$$
\eta_{i}(t)=\frac{R^{\omega}(t)-R(t)}{c_{i}}
$$

式中: $\eta_{i}(t)$ 为第 $i$ 个组件的成本效益, 定义为单位 成本提升的系统可靠度增量; $R(t)$ 为第 $i$ 个组件更 换之前的系统可靠度函数; $R^{\omega}(t)$ 为第 $i$ 个组件更换 之后的系统可靠度函数; 第 $i$ 个组件更换后对整个 系统的可靠度会有一个提升量, 此时的系统可靠度 函数可以表示为 $R^{\omega}(t)$, 且 $R^{\omega}(t)<1$ 。

为了量化描述 $R^{\omega}(t)$, 首先要开展第 $i$ 个组件的 更换行为对第 $i$ 个组件可靠度的影响研究。假设第 $i$ 个组件工作一段时间后对其进行更换, 此时的组件 可靠度相当于通过一个时间的正向偏移量来提升其 可靠度直到为 1 , 即

$$
R_{i}^{\omega}(t)=R_{i}(t-\tau)
$$

式中: $\tau$ 为在更换时刻将第 $i$ 个组件可靠度提升到 1 时所需的时间正向偏移量 $; R_{i}^{\omega}(t)$ 为第 $i$ 个组件更换 之后的组件可靠度函数。

由此可知, $R^{\omega}(t)$ 可以表示为 $R^{\omega}(t)=R(t-\varepsilon)$, $0<\varepsilon<\tau$, 其中 $\varepsilon$ 表示第 $i$ 个组件进行更换后对整 个系统可靠度提升所需的时间正向偏移量。

为了使 $R(t)$ 能够由含 $R_{i}(t)$ 的表达式来表示, 采用枢轴分解定理 ${ }^{[6]}$ 和 $\mathrm{BI}$ 理论 ${ }^{[7]}$ 对 $R(t)$ 进行变形 展开,具体过程如下。

由枢轴分解定理可知, $R(t)$ 可以表示为

$$
R(t)=R_{i}(t) R\left(1_{i}, t\right)+\left(1-R_{i}(t)\right) R\left(0_{i}, t\right)(6)
$$
式中: $R_{i}(t)$ 为第 $i$ 个组件的可靠度; $R\left(1_{i}, t\right)$ 为当第 $i$ 个组件正常时在 $t$ 时刻的系统可靠度; $R\left(0_{i}, t\right)$ 为当 第 $i$ 个组件失效时在 $t$ 时刻的系统可靠度。

根据 BI 理论, 第 $i$ 个组件在 $t$ 时刻的重要度 $I_{B}^{i}$ 可 以表示为

$$
I_{B}^{i}(t)=\frac{\partial R(t)}{\partial R_{i}(t)}=R\left(1_{i}, t\right)-R\left(0_{i}, t\right)
$$

将 (7) 式代人 $(6)$ 式可知, $R(t)$ 可以变形为

$$
R(t)=R_{i}(t) I_{B}^{i}(t)+R\left(0_{i}, t\right)
$$

同理可知, $R^{\omega}(t)$ 可以变形为

$$
R^{\omega}(t)=R_{i}^{\omega}(t) I_{B}^{i}(t)+R\left(0_{i}, t\right)
$$

将(8) 式和(9) 式代人(4) 式可得

$$
\eta_{i}(t)=\frac{\left(R_{i}^{\omega}(t)-R_{i}(t)\right) I_{B}^{i}(t)}{c_{i}}
$$

定义成本效益重要度 $I_{C}^{i}(t)$ 为第 $i$ 个组件更换 后的成本效益 $\eta_{i}(t)$ 与每个组件更换后的成本效益 之和 $\sum_{i=1}^{n} \eta_{i}(t)$ 的比值, 也就是说 $I_{C}^{i}(t)$ 为一个经归一 化的值, 即

$$
I_{C}^{i}(t)=\frac{\eta_{i}(t)}{\sum_{i=1}^{n} \eta_{i}(t)}=\frac{\frac{\left(R_{i}^{\omega}(t)-R_{i}(t)\right) I_{B}^{i}(t)}{c_{i}}}{\sum_{i=1}^{n} \frac{\left(R_{i}^{\omega}(t)-R_{i}(t)\right) I_{B}^{i}(t)}{c_{i}}}
$$

\section{3 基于成本效益重要度的优化策略}

在系统可靠度不低于预设國值以及运行时间达 到设定时间 2 个条件下,基于成本效益重要度的优 化策略,采用模拟消耗式组件的方式来反向求解各 组件的最优更换序列。当系统可靠度低于预设阈值 时,将待更换的各组件成本效益重要度从大到小依 次排列, 对成本效益重要度最大的组件进行更换。 假设更换过程没有时间延迟，更换完成后系统继续 运行, 并根据约束条件决策是否进人下一次更换。 最后, 求出整个保障周期内各组件的更换时刻,形成 最优的组件更换序列。详细的组件更换序列优化过 程如图 2 所示。

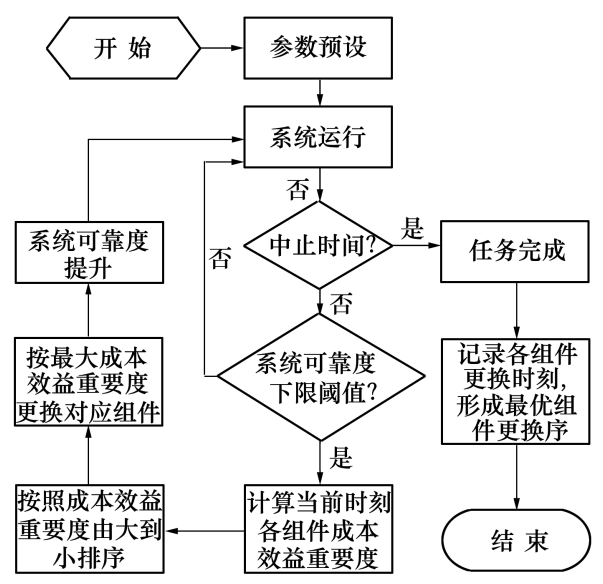

图 2 组件更换序列优化过程

步骤 1 参数预设。按照任务的要求, 设置系 统可靠度下限阈值以及运行时间。

步骤 2 系统运行。判断系统运行时间是否到 达任务中止时间, 如果到达, 任务完成; 如未到达, 判 
断系统可靠度是否下降到预设阈值。如果高于预设 阈值, 系统继续运行; 如果低于预设阈值, 进行步 骤 3 ;

步骤 3 组件更换。当系统可靠度下降到下限 阈值时, 计算各组件成本效益重要度, 取最大成本效 益重要度对应的组件进行更换, 其形式化见 (12) 式,更换完成后, 转到步骤 2 继续运行。

$$
\begin{aligned}
& c_{s_{1}}=f_{1}\left(\sigma, S, \max \left\{I_{C}^{1}, I_{C}^{2}, \cdots, I_{C}^{i}, \cdots, I_{C}^{n}\right\}\right) \\
& c_{s_{2}}=f_{2}\left(\sigma, S, \max \left\{I_{C}^{1}, I_{C}^{2}, \cdots, I_{C}^{i}, \cdots, I_{C}^{n}\right\}\right) \\
& \vdots \\
& c_{s_{j}}=f_{j}\left(\sigma, S, \max \left\{I_{C}^{1}, I_{C}^{2}, \cdots, I_{C}^{i}, \cdots, I_{C}^{n}\right\}\right) \\
& \vdots \\
& c_{s_{k}}=f_{k}\left(\sigma, S, \max \left\{I_{C}^{1}, I_{C}^{2}, \cdots, I_{C}^{i}, \cdots, I_{C}^{n}\right\}\right)
\end{aligned}
$$

式中: $c_{s_{j}}$ 为第 $j$ 次更换时刻需要更换的组件; $f_{j}(\cdot)$ 为 第 $j$ 次更换时刻的映射函数。

步骤 4 形成最优组件更换序列。当任务完成 后, 将各组件更换的时刻进行记录, 形成最优组件更 换序列，即 $\left\{c_{s_{1}}, c_{s_{2}}, \cdots, c_{s_{j}}, \cdots, c_{s_{k}}\right\}$ 。

\section{4 数值实例验证}

\section{1 系统规格说明}

以一个由 6 个组件构成的串并联系统为例说明 系统可靠性的建模过程, 如图 3 所示。

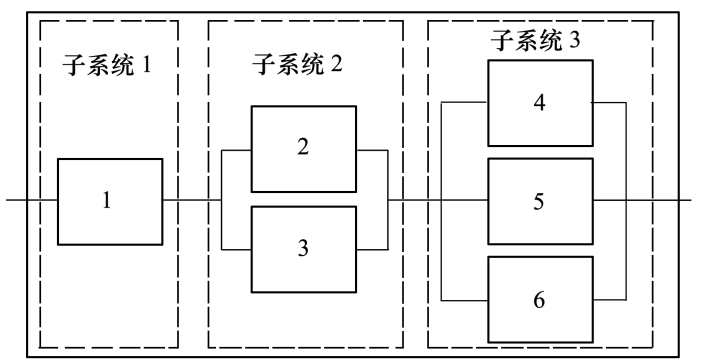

图 3 由 6 个组件构成的串并联系统

由(2) 式可知, 图 3 中系统可靠度为

$$
\begin{array}{r}
R(t)=R_{1}(t) \cdot\left(1-\left(1-R_{2}(t)\right) \cdot\left(1-R_{3}(t)\right)\right) \cdot \\
\quad\left(1-\left(1-R_{4}(t)\right) \cdot\left(1-R_{5}(t)\right) \cdot\left(1-R_{6}(t)\right)\right)
\end{array}
$$

许多系统失效都可以追溯到潜在的退化, 例如 微机电系统中组件摩擦表面上的磨损遵循线性退化 路径。在微机电系统中, 该线性模型已被用于表征 磨损过程的失效机理。根据文献 [14-15], 总结的组
件规格参数值如表 1 所示。假设运行中涉及的参数 如表 2 所示。

表 1 组件规格参数值

\begin{tabular}{ccccc}
\hline$i$ & $\mu_{i}$ & $\varepsilon_{i}$ & $L_{i}$ & $c_{i}$ \\
\hline 1 & 1.06 & $N(0,1)$ & 10 & 0.40 \\
2 & 1.16 & $N(0,1)$ & 8 & 0.20 \\
3 & 1.20 & $N(0,2)$ & 10 & 0.30 \\
4 & 1.18 & $N(0,2)$ & 9 & 0.18 \\
5 & 1.20 & $N(0,3)$ & 9 & 0.15 \\
6 & 1.10 & $N(0,3)$ & 10 & 0.35 \\
\hline
\end{tabular}

表 2 运行参数

\begin{tabular}{ccc}
\hline 参数 & 参数值 & 说明 \\
\hline$S /$ 月 & 16 & 运行总周期 \\
$\sigma$ & 0.90 & 系统可靠度下限阈值 \\
\hline
\end{tabular}

\section{2 策略验证}

设定系统可靠度下限阈值 $\sigma=0.90$ 和任务运行 时间 $S=16$, 根据所提系统保障策略进行各组件更 换时刻的优化过程。当系统第一次运行时, 随着时 间的进行, 系统的可靠度逐渐下降, 直到系统可靠度 低于 $\sigma$ 时,即当 $t=7.08$ 时, 按照(11) 式计算当前时 刻的成本效益重要度, 即 $I_{C}^{2}=0.4716, I_{C}^{3}=0.3144$, $I_{C}^{5}=0.0874, I_{C}^{4}=0.0728, I_{C}^{6}=0.0375, I_{C}^{1}=0.0163$ 。 从成本效益重要度值表明: 成本效益重要度越大产 生的效益越大; 成本效益重要度趋近于 0 时表示不 产生效益。因此, 选择成本效益重要度最大值对应 的组件进行更换, 即选择第 2 个组件进行更换, 更换 完成后, 系统可靠度提升至 0.9812 , 此时产生了新 系统。在新系统的基础上进行下一次迭代过程, 以 此类推,直到到达任务中止时间。系统组件更换明 细如表 3 所示, 在整个运行周期内的系统可靠度变 化规律如图 4 所示。

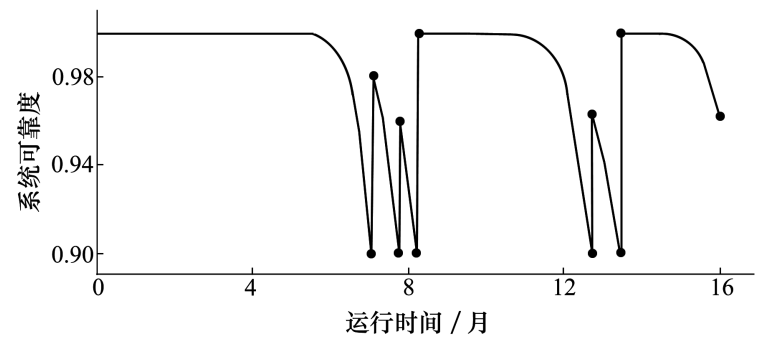

图 4 系统可靠度随时间变化规律 
表 3 系统组件更换明细

\begin{tabular}{cccccc}
\hline $\begin{array}{c}\text { 更换 } \\
\text { 次数 }\end{array}$ & $\begin{array}{c}\text { 系统 } \\
\text { 可靠度 }\end{array}$ \\
\hline 1 & 0.8988 & 7.08 & $I_{C}^{2}=0.4716, I_{C}^{3}=0.3144, I_{C}^{5}=0.0874, I_{C}^{4}=0.0728, I_{C}^{6}=0.0375, I_{C}^{1}=0.0163$ & 0.9812 & $\begin{array}{c}\text { 提时升后系 需更换的 } \\
\text { 统可靠度 组件编号 }\end{array}$ \\
2 & 0.8984 & 7.78 & $I_{C}^{5}=0.4020, I_{C}^{4}=0.3350, I_{C}^{6}=0.1723, I_{C}^{1}=0.0908, I_{C}^{2}=0, I_{C}^{3}=0$ & 0.9602 & 5 \\
3 & 0.8991 & 8.23 & $I_{C}^{1}=1, I_{C}^{2}=0, I_{C}^{3}=0, I_{C}^{4}=0, I_{C}^{5}=0, I_{C}^{6}=0$ & 1 & 1 \\
4 & 0.8987 & 12.69 & $I_{C}^{2}=0.3111, I_{C}^{5}=0.2128, I_{C}^{3}=0.2074, I_{C}^{4}=0.1774, I_{C}^{6}=0.0912, I_{C}^{1}=0$ & 0.9640 & 2 \\
5 & 0.8991 & 13.44 & $I_{C}^{5}=0.4421, I_{C}^{4}=0.3684, I_{C}^{6}=0.1895, I_{C}^{1}=0, I_{C}^{2}=0, I_{C}^{3}=0$ & 1 & 5 \\
6 & 0.8981 & $S<16.46$ & &
\end{tabular}

注: 提升后系统可靠度为 1 表示无限接近 1 。

根据仿真结果可知: (1)在整个运行周期内, 各组 件最优更换序列为 $\{(7.08,2),(7.78,5),(8.23,1)$, $(12.69,2),(13.44,5)\}$; 2)系统在时刻 7.08,7.78, $8.23,12.69,13.44$ 等五处分别进行了组件更换工 作, 更换之后系统的可靠度有了明显提升; 3在时刻 7.78 处, 对选择的组件更换后系统可靠度提升量较 小是因为: $I_{C}^{5}$ 虽然值最大, 但与 $I_{C}^{4}$ 相比差异不大。 由于限定每次仅能更换一个组件, 因此 $I_{C}^{5}$ 对系统可 靠度提升的贡献度相对较小; (4) 对于当前系统来说, 1 号、2 号、5 号组件为瓶颈节点。

\section{5 结 论}

针对多组件退化复杂系统, 提出了一种基于成 本效益重要度的复杂系统可靠性保障策略。根据成 本效益重要度最大值选择系统中最需要被更换的组 件, 基于成本效益重要度选择组件的方法有助于识 别系统的瓶颈并防止系统发生意外失效。此外,所 提策略不仅可以识别系统中最需要更换的组件, 而 且在一次运行周期内使系统维护成本达到最小。数 值实例验证了所提策略的可行性。

\section{参考文献:}

[1] AHMAD R, KAmarudDin S. An Overview of Time-Based and Condition-Based Maintenance in Industrial Application [J]. Computers \& Industrial Engineering, 2012, 63(1):135-149

[2] JARDine A K S, TSANG A H C. Maintenance, Replacement, and Reliability: Theory and Applications [M]. Boca Raton: Taylor \& Francis Group, 2013

[3] WANG W, WANG H. Preventive Replacement for Systems with Condition Monitoring and Additional Manual Inspection[J]. European Journal of Operational Research, 2015, 247(2): 459-471

[4] YUN W Y, ENDharta A J. A Preventive Replacement Policy Based on System Critical Condition[J]. Journal of Risk and Reliability, 2016, 230(1): 93-100

[5] WANG C, XU J, WANG H, et al. A Criticality Importance-Based Spare Ordering Policy of Multi-Component Degraded Systems [J]. Eksploatacja i Niezawodnosc-Maintenance and Reliability, 2018, 20(4) : 662-670

[6] KUO W, ZHU X. Importance Measures in Reliability, Risk, and Optimization: Principles and Applications [M]. Chichester: Wiley, 2012

[7] BIRNBAuM Z W. On the Importance of Different Components in a Multicomponent System: in Multivariate Analysis [M]. San Diego: Academic Press, 1969

[8] KUO W, ZUO M J. Optimal Reliability Modeling: Principles and Application[M]. New York: John Wiley \& Sons, 2003

[9] SI S, DUI H, ZHAO X, et al. Integrated Importance Measure of Component States Based on the Loss of the Performance[ J]. IEEE Trans on Reliability, 2012, 61(1) : 192-202 
[10] WU S, COOLEN F. A Cost-Based Importance Measure for System Components: an Extension of the Birnbaum Importance[J]. European Journal of Operational Research, 2013, 225(1): 189-195

[11] 杜永军, 郭雅琪, 蔡志强, 等. 基于混合 C-谱的 K-终端网络置换重要度计算方法 [J]. 西北工业大学学报, 2019, 37(5): 897-902

DU Yongjun, GUO Yaqi, CAI Zhiqiang, et al. K-Terminal Network Permutation Importance Measure Based on Mixture C-Spectrum [J]. Journal of Northwestern Polytechnical University, 2019, 37(5) : 897-902 (in Chinese)

[12] LYU D, SI S. Dynamic Importance Measure for the K-out-of-N: G System under Repeated Random Load[J]. Reliability Engineering \& System Safety, 2020, 195: 106720

[13] LIU B, LIANG Z, PARLIKAD A K, et al. Condition-Based Maintenance for Systems with Aging and Cumulative Damage Based on Proportional Hazards Model[J]. Reliability Engineering \& System Safety, 2017, 168:200-209

[14] PENG H, FENG Q, COIT D W. Simultaneous Quality and Reliability Optimization for Microengines Subject to Degradation[J]. IEEE Trans on Reliability, 2009, 58(1): 98-105

[15] PENG H, COIT D W, FENG Q. Component Reliability Criticality or Importance Measures for Systems with Degrading Components $[\mathrm{J}]$. IEEE Trans on Reliability, 2012, 61(1): 4-12

\title{
Reliability Guarantee Strategy for Complex System Based on Cost-Benefit Importance
}

\author{
WANG Cheng ${ }^{1}$, XU Jianxin ${ }^{2}$, ZHANG Zhenming ${ }^{2}$, WANG Hongjun ${ }^{3}$ \\ 1.School of Mechanical Engineering, Xi'an Aeronautical University, Xi' an 710077, China; \\ 2.School of Mechanical Engineering, Northwestern Polytechnical University, Xi'an 710072, China; \\ 3. Key Laboratory of Modern Measurement and Control Technology of Ministry of Education, \\ Beijing Information Science and Technology University, Beijing 100192, China
}

\begin{abstract}
In order to ensure the long-term stable and economic operation of complex system, the system operation process is described and the problems is solved, and a system reliability model and an optimization model for component replacement are constructed. Based on the theory of marginal utility and importance measures, a reliability guarantee strategy for complex system based on cost-benefit importance is presented, which aims to find a component preventive replacement sequence with minimum maintenance cost on the constraints of system reliability lower threshold and running time. When the system reliability drops to a preset threshold, the cost-benefit importance of each component is calculated, the component with the greatest cost-benefit importance to replace is selected, and then iterate until the operation task is completed to form an optimal component replacement sequence. The feasibility of the present strategy is verified by taking a complex system which can be equivalent to a series-parallel system as an example. The present strategy has certain reference significance of ensuring the reliable operation of some high-end equipment safety-critical systems.
\end{abstract}

Keywords : complex system; reliability guarantee; cost-benefit importance; marginal utility; preventive replacement 